\title{
Osteoarthritis: a holistic approach
}

\author{
Andrew Barr and Philip G Conaghan
}

\begin{abstract}
Osteoarthritis represents a massive and rapidly increasing burden on our society and the NHS. Current treatments are limited in efficacy and have significant toxicities. A conference was organised in conjunction with the British Society of Rheumatology with the aim of updating frontline clinicians and researchers on the size and causes of this problem, with a focus on modern management.
\end{abstract}

KEY WORDS: osteoarthritis, treatment, assessment, chronic pain

\section{The burden of osteoarthritis}

Osteoarthritis (OA) is the most common form of arthritis and for affected individuals is characterised by pain, joint dysfunction and reduced quality of life. The symptomatic prevalence in the adult population has been estimated at $9 \%$, which makes it almost 20 -fold more common than rheumatoid arthritis $(0.5 \%)$ and gout $(0.5 \%){ }^{1}$

Osteoarthritis therefore represents a significant workload for primary care services. In England in 2009-10, the estimated number of people who consulted a GP and were assigned a code of OA was 800,000 (derived from North Staffordshire general practice databases). Similarly, the number of hospital admissions with a primary diagnosis of OA in 2009-10 was 221,000, indicating a similar burden on secondary care. ${ }^{2}$

People with OA have a higher risk of death compared with the general population. ${ }^{3}$ Age and obesity are important determinants in OA. In the context of an aging and increasingly obese population, the need for prevention and novel effective treatment strategies is of vital importance. The burden of OA is compounded by the relatively small effect size of current non-surgical therapy.

\section{Aetiopathogenesis}

Healthy cartilage matrix consists of type II collagen fibres, which confer tensile strength, along with proteoglycan (aggrecan), which draws water into the tissue to provide shock absorption. Relatively small numbers of chondrocytes

\footnotetext{
Andrew Barr, ${ }^{1}$ specialist registrar in rheumatology; and Philip G Conaghan, ${ }^{2,3}$ professor of musculoskeletal medicine

${ }^{1}$ Hammersmith Hospital, Imperial College NHS Trust, London; ${ }^{2}$ Section of Musculoskeletal Disease, Leeds Institute of Molecular Medicine, University of Leeds; ${ }^{3}$ NIHR Leeds Musculoskeletal Biomedical Research Unit, Chapel Allerton Hospital, Leeds
}

maintain this large matrix. Osteoarthritis is characterised by a failure to repair damaged cartilage but also by subchondral bone changes due to biomechanical and biochemical changes in the joint tissues, which are, to some extent, age related. The reparative anabolic processes of the matrix chondrocytes are replaced by an excess of catabolic processes driven by proteases including aggrecanases and collagenases. The consequence is a more fragile cartilage, with perhaps stiffer subchondral bone, and a vicious biomechanical and biochemical cycle ensues.

People typically present with established multi-tissue disease and hence early disease is poorly defined. Preclinical animal models may provide insight into early pathogenesis and some of the connections between the biomechanical and biochemical changes. For example, the induction and expression of genes that encode the catabolic proteases seem to be mechanosensitive. Some of these genes have greater expression in weight-bearing joints and reduced expression in nonweight-bearing joints in mouse models of joint injury. This concept that cartilage catabolism in OA may depend upon weight bearing or joint loading is supported by cartilage regeneration occurring following surgical unloading of human joints. ${ }^{4}$

Osteoarthritis in humans represents a heterogeneous pathologic process with many potential therapeutic targets. It is therefore unlikely that a single 'magic bullet' will effectively treat all patients.

\section{Osteoarthritis treatment}

The European League Against Rheumatism (EULAR), Osteoarthritis Research Society International (OARSI) and National Institute for Health and Clinical Excellence (NICE) provide evidence-based guidelines for the treatment of $\mathrm{OA}$ (Table 1). There is international consensus on the need for a holistic assessment of people with OA, and therapy with a combination of non-pharmacological and pharmacological therapies, before considering surgery.

\section{A holistic approach}

The impact of OA on an individual's function, quality of life, mood, relationships, occupation and leisure activities may be extensive. Pain has many determinants and can be influenced by comorbidities including mood disorders, loneliness and sleeping problems. Applying a biopsychosocial model of care encompasses these needs and may include personalised selfmanagement strategies and education to encourage active 


\begin{tabular}{|c|c|c|c|c|}
\hline \multirow{2}{*}{ Therapy } & \multicolumn{4}{|c|}{ Guideline (site of OA) $\dagger$} \\
\hline & $\begin{array}{l}\text { EULAR } \\
\text { (hand) }\end{array}$ & $\begin{array}{l}\text { NICE } \\
\text { (all sites) }\end{array}$ & $\begin{array}{l}\text { OARSI } \\
\text { (hip) }\end{array}$ & (knee) \\
\hline Exercise & + & + & + & + \\
\hline Education & + & + & + & + \\
\hline Weight loss & nil & + & + & + \\
\hline $\begin{array}{l}\text { Thermotherapy } \\
\text { (eg hot packs) }\end{array}$ & + & + & ? & $?$ \\
\hline $\begin{array}{l}\text { Aids, braces and } \\
\text { footwear (site specific) }\end{array}$ & + & + & + & + \\
\hline Acupuncture & nil & ? & nil & ? \\
\hline Paracetamol & + & + & + & + \\
\hline Topical NSAIDs & + & + & nil & + \\
\hline $\begin{array}{l}\text { Oral NSAIDs (lowest } \\
\text { possible dose) }\end{array}$ & + & + & + & + \\
\hline $\begin{array}{l}\text { Opioids (for } \\
\text { refractory pain) }\end{array}$ & nil & + & + & + \\
\hline $\begin{array}{l}\text { Glucosamine and } \\
\text { chondroitin sulphate }\end{array}$ & ? & - & nil & ? \\
\hline $\begin{array}{l}\text { Intra-articular } \\
\text { corticosteroids }\end{array}$ & + & + & + & + \\
\hline $\begin{array}{l}\text { Intra-articular } \\
\text { hyaluronans }\end{array}$ & ? & - & ? & ? \\
\hline \multicolumn{5}{|l|}{ Surgery } \\
\hline $\begin{array}{l}\text { Lavage/ } \\
\text { debridement }\end{array}$ & nil & - & nil & $?$ \\
\hline $\begin{array}{l}\text { TJR/arthroplasty } \\
\text { (site specific) }\end{array}$ & + & + & + & + \\
\hline
\end{tabular}

$+=$ treatment recommended; ? = treatment may be useful;

$-=$ treatment not recommended; EULAR = European League Against Rheumatism; NICE = National Institute for Health and Clinical Excellence; nil = treatment opinion not provided; NSAID = non-steroidal antiinflammatory drug; OARSI = Osteoarthritis Research Society International; $\mathrm{TJR}=$ total joint replacement.

*This is not a head-to-head comparison of the guidelines but a summary of the recommendations. †Each guideline addresses different anatomical sites.

participation in treatment and positive behavioural changes. These are considered to be essential components of chronic disease management.

\section{Non-pharmacological therapies}

Although these treatment strategies work well in combination, the most important is probably exercise. The aim of exercise is to unload damaged joints, which is of particular importance in this mechanically driven disease. Effective reductions in pain and disability in patients with OA of the knee have been identified by a meta-analysis of aerobic walking and home-based quadriceps-strengthening exercises. ${ }^{5}$ The effect size of aerobic knee exercises in OA is moderate. ${ }^{6}$
Self-management, patient education and lifestyle modification are important objectives of treatment. However, the individual effect sizes of these interventions are small. ${ }^{6}$

\section{Pharmacological therapies}

When considering pharmacological therapies for a person with $\mathrm{OA}$, the properties of the drug should be considered, including its efficacy, toxicity, dosing regimen and cost. These should be interpreted in the context of the patient's age, current medications, likely compliance and concurrent gastrointestinal and cardiovascular risks.

The use of topical non-steroidal anti-inflammatory drug (NSAID) therapy, instead of oral therapy, confers less systemic toxicity and comparable pain relief when used regularly. ${ }^{7}$ However, the need for multiple applications may result in poor compliance. Traditional oral NSAIDs and cyclooxygenase 2 (COX-2) selective inhibitors ideally should be prescribed for a short time period to limit toxicity. Both classes share dosedependent effects on cardiovascular risk (with the possible exception of naproxen), but COX-2 inhibitors confer a lower gastrointestinal risk. ${ }^{8}$

The use of opioids in OA has been reported to have a moderate effect on pain control, ${ }^{6}$ but a smaller effect on function; trials have often been of short duration. Although side effects such as drowsiness and constipation are well known, a recent retrospective study (in patients with a mean age of 81 years) identified a significantly greater risk of fracture after initiating opioids compared with NSAIDs. This risk was increased for the first two weeks and not thereafter. Shortacting opioids were also associated with a greater risk of fracture. ${ }^{9}$

\section{Surgical treatments}

Total joint replacement in OA is intended for patients with joint symptoms that have a substantial impact on quality of life and that are refractory to non-surgical treatment. According to NICE, decisions regarding the referral threshold should be based on discussions between patient representatives, referring clinicians and surgeons rather than the results of priority scoring tools. Such referrals should be timely to avoid prolonged and established functional limitation with severe pain. Referral of a patient for joint replacement should not be precluded by increasing age, obesity or comorbidities. ${ }^{10}$ Other invasive treatments for OA of the knee, such as arthroscopic lavage and debridement, have specific indications. These should be offered to those with a clear history of mechanical locking. However, there is no benefit from using these therapies in patients with knee gelling, 'giving way' or radiographic evidence of loose bodies. ${ }^{10}$

\section{Chronic pain in osteoarthritis and therapeutic strategies}

The chronic pain of OA can be severe and nocturnal and may differ from acute pain because of sensitisation. The enhanced 
pain experienced in response to a given stimulus reflects neural plasticity.

In acute pain, a peripheral nociceptor transduces a stimulus into an action potential in an afferent sensory neurone that relays with a spinal neurone. This transmits the signal on to the thalamus and then the sensory cortex. Within the central nervous system, serotonergic and noradrenergic pathways provide descending inhibition to modulate and reduce the signal that is conveying the pain. In chronic pain, this signal can be enhanced by:

- greater sensitivity in the peripheral nociceptor (peripheral sensitisation)

- greater transmission from the spine to the cortex due to inhibition of the inhibitory central descending pathways (central sensitisation)

- altered spontaneous and evoked activity from peripheral neuronal injury (neuropathic pain).

Amitriptyline has historically been used to address central sensitisation. However, when duloxetine, a serotonin and noradrenaline reuptake inhibitor, was used in a 13-week randomised controlled trial, significant benefits in pain and functional scores were reported in patients with OA of the knee. ${ }^{11}$ Further studies are necessary to establish its long-term clinical utility.

One of the major mediators of peripheral sensitisation and pain in animal models of OA is nerve growth factor (NGF). Blockade of the NGF mechanism with the monoclonal antibody tanezumab in a randomised controlled trial in humans demonstrated a sustained improvement in knee pain and was essentially well tolerated. However, ongoing trials have been suspended and this class continues to be investigated due to increased rates of joint replacement. ${ }^{12}$

A further example of neural plasticity is the placebo effect. This is where a biological effect may occur in response to the way in which the patient interprets their medical experience or environment (a meaning response) rather than in response to an active treatment that directly induces this effect. The size of the placebo analgesia effect of randomised control trials in patients with OA can be moderate to large and is believed to be facilitated by descending inhibition of afferent nociceptive pathways. Some of the most powerful placebo effects are created by spending greater time with the patient, personalising care and ensuring follow up. In the absence of particularly effective therapies for $\mathrm{OA}$, it may be reasonable to employ these approaches in daily clinical practice to improve pain and quality of life.

\section{Conclusions}

Osteoarthritis represents a heavy burden for individuals and health services. It is an increasingly prevalent, painful and debil- itating condition that is, in part, driven by an obese and aging population. An international consensus exists on the treatment strategies in OA, but the effect size of many treatments is small. These therapies should be judiciously combined in the context of the patient's needs and comorbidities and benefit-risk assessments to provide optimal individual therapy. Identifying specific patient phenotypes with shared underlying pathogenesis may improve the use of symptom-modifying therapies and even permit the development of disease-modifying therapies.

\section{References}

1 Salaffi F, De Angelis R, Grassi W. Prevalence of musculoskeletal conditions in an Italian population sample: results of a regional community-based study. I. The MAPPING study. Clin Exp Rheumatol 2005;23:819-28.

2 Hospital Episode Statistics Online. ICD10: M15-M19 (2009-2010). http://www.hesonline.nhs.uk/Ease/servlet/ContentServer?siteID=1937 \&categoryID=192 [Accessed 27 January 2012].

3 Nüesch E, Dieppe P, Reichenbach S et al. All cause and disease specific mortality in patients with knee or hip osteoarthritis: population based cohort study. BMJ 2011;342:d1165.

4 Intema F, Van Roermund PM, Marijnissen AC et al. Tissue structure modification in knee osteoarthritis by use of joint distraction: an open 1-year pilot study. Ann Rheum Dis 2011;70:1441-6.

5 Roddy E, Zhang W, Doherty M. Aerobic walking or strengthening exercise for osteoarthritis of the knee? A systematic review. Ann Rheum Dis 2005;64:544-8.

6 Zhang W, Nuki G, Moskowitz RW et al. OARSI recommendations for the management of hip and knee osteoarthritis: part III: changes in evidence following systematic cumulative update of research published through January 2009. Osteoarthritis Cartilage 2010;18:476-99.

7 Underwood M, Ashby D, Cross P et al. Advice to use topical or oral ibuprofen for chronic knee pain in older people: randomised controlled trial and patient preference study. BMJ 2008;336:138-42.

8 Warner TD, Mitchell JA. COX-2 selectivity alone does not define the cardiovascular risks associated with non-steroidal anti-inflammatory drugs. Lancet 2008;371:270-3.

9 Miller M, Stürmer T, Azrael D et al. Opioid analgesics and the risk of fractures in older adults with arthritis. J Am Geriatr Soc 2011;59:430-8.

10 Conaghan PG, Dickson J, Grant RL. Care and management of osteoarthritis in adults: summary of NICE guidance. BMJ 2008;336:502-3.

11 Chappell AS, Ossanna MJ, Liu-Seifert H et al. Duloxetine, a centrally acting analgesic, in the treatment of patients with osteoarthritis knee pain: a 13-week, randomized, placebo-controlled trial. Pain 2009;146:253-60.

12 Lane NE, Schnitzer TJ, Birbara CA et al. Tanezumab for the treatment of pain from osteoarthritis of the knee. $N$ Engl J Med 2010;363:1521-31.

Address for correspondence: Andrew Barr, Rheumatology Department, Hammersmith Hospital, Imperial NHS Trust, Du Cane Road, London W12 0HS. Email: barrandrew@aol.com 